Punjab University Journal of Mathematics (2021),53(7),511-526

https://doi.org/10.52280/pujm.2021.530705

\title{
Theory of Bijective Hypersoft Set with Application in Decision Making
}

\author{
Atiqe Ur Rahman ${ }^{1}$, Muhammad Saeed ${ }^{2, *}$, Abida Hafeez ${ }^{3}$ \\ $1,2,3$ Department of Mathematics, \\ University of Management and Technology, Lahore, Pakistan, \\ Email: aurkhb@gmail.com ${ }^{1}$, muhammad.saeed@umt.edu.pk ${ }^{2, *}$, \\ f2019109046@umt.edu.pk ${ }^{3}$
}

Received: 06 March,2021 / Accepted: 17 June, 2021 / Published online:27 July, 2021

\begin{abstract}
Hypersoft set (an extension of soft set) is a new mathematical tool to tackle the inadequacy of soft set for attribute-valued sets. In this study, concept of bijective hypersoft set is proposed and some of its set theoretic operations like restricted-AND and relaxed-AND, are characterized. Moreover, new operations of bijective hypersoft set such as dependency, decision system, significance of decision system, reduced decision system and decision rules in decision system, are discussed with illustrated examples. A decision making algorithm and application are discussed with the support of these proposed operations.
\end{abstract}

\section{AMS (MOS) Subject Classification Codes: 91B06; 93E25}

Key Words: Soft set, Bijective soft set, Hypersoft set, Bijective hypersoft set, Bijective hypersoft decision system.

\section{INTRODUCTION}

In 1999, Molodtsov [19] developed a new structure known as soft set in literature to address the shortcoming of the existing structures (i.e. fuzzy set, intuitionistic fuzzy etc.) dealing with uncertain and vague data of information. He provided a strong basis for parameterized interpretation of hesitant information through the introduction of this structure. Later on, Maji et al. [17] investigated different elementary essentials i.e. properties, set theoretic operations, laws and results of soft set for its further implementation in certain disciplines. In 2005, Pei et al. [22] illustrated the relational concept between soft sets and information systems. They confirmed the validity of the soft set as a parameterized family of special information system. The researchers Ali et al. [2], Babitha et al. [3, 4], Sezgin et al. [39], Ge et al. [6] and Li [16] studied soft set professionally and extended the concept with the introduction of certain new features i.e. restrictedness on operations, set relation and function. Saeed et al. [29] explored the concept of soft elements and soft members under soft set environment. Kamaci et al. [9, 10, 11, 12] developed bijective soft matrix theory with multi-bijective linguistic soft decision, investigated difference operations of soft matrices with applications in decision making system, introduced $N$-soft algebraic structures, 
and discussed bipolar $N$-soft set theory with applications respectively. Petchimuthu et al. [23] made valuable discussion on the row-products of inverse soft matrices with application in multicriteria decision making. Many researchers [14, 5, 15, 21, 1, 43] expanded soft set theory with the development of soft-like hybrids to have useful results as well as utilization in decision making problems. Gong et al. [7] introduced the concept of bijective soft set and investigated its some properties.

In 2018, Smarandache [40] developed a new structure hypersoft set (HSS) to adequate the soft set for multi attribute-valued functions. In 2020, Saeed et al. [30, 31] characterized the necessary basic axioms, properties, laws and set theoretic operations of HSS with the help of appropriate examples. In 2020, Rahman et al. [24, 25] enhanced the study of HSS to develop fuzzy-like structures with complex sets and also studied certain properties of convexity under HSS-environment. In 2021, Rahman et al. [26, 27, 28] studied decision making applications based on neutrosophic parameterized hypersoft Set, fuzzy parameterized hypersoft set and rough hypersoft set. Saeed et al. [32, 33, 34, 35] discussed decision making techniques for mappings on hypersoft classes, neutrosophic hypersoft mapping and complex multi-fuzzy hypersoft set. They also developed hypersoft graphs with some properties. Ihsan et al. [8] investigated hypersoft expert set with application in decision making for the best selection of product. Yolcu et al. [41, 42] conceptualized the theories of fuzzy and intuitionistic fuzzy hypersoft sets with their employment in decision making. Ozturk et al. [20] introduced neutrosophic hypersoft topological spaces and discussed their important properties. Saqlain et al. $[36,37,38]$ developed single and multi-valued neutrosophic hypersoft sets along with calculation of tangent similarity measure of single valued neutrosophic hypersoft sets, characterized aggregate operators of neutrosophic hypersoft set and employed TOPSIS method for neutrosophic hypersoft sets using accuracy function with application. Martin et al. [18] investigated concentric plithogenic hypergraph based on plithogenic hypersoft sets. Kamac1 [13] made very valuable research on the hybrid structures of hypersoft sets and rough sets.

In many real life situations, distinct attributes are further partitioned into disjoint attributevalued sets. Decision makers may suffer some kind of inclination and penchant while ignoring such partitioning of attributes during the judgment. The existing soft set theory is not projected for such sets. Therefore a new structure demands its place in literature for addressing such impediment, so hypersoft set theory is conceptualized to tackle such situations. This novel structure has increased the flexibility and reliability of decision making process. It not only addresses the inadequacy of existing soft-like structures for multiargument approximate functions but also helps the decision makers to decide the matters with deep observation. In this study, a new type of hypersoft set, bijective hypersoft set (BHSS), is characterized which assigns the disjoint approximate sets to each tuple in the cartesian product of attribute-valued sets. Moreover, certain elementary properties are investigated with supporting examples. A decision system is developed based on set theoretic operations of BHSS and is applied in decision making with illustrated example.

The rest of the paper is organized as:

In section 2, some fundamental definitions and terms are recalled from already published relevant literature, section 3 formulates the theory of bijective hypersoft set and its decision system, section 4 proposes a decision-making algorithm with application for the best 
selection of an appropriate product and finally, section 5 concludes the paper with future directions.

\section{PRELIMINARIES}

In this section, we present some basic terminologies for proper understanding of the proposed work. Throughout the paper, $\mathfrak{U}$ denotes the universe of discourse.

Definition 1. [19]

A pair $\left(\zeta_{S}, \Lambda\right)$ is called a soft set over $\mathfrak{U}$, where $\zeta_{S}: \Lambda \rightarrow \mathcal{P}(\mathfrak{U})$ and $\Lambda$ be a set of attributes of $\mathfrak{U}$.

Definition 2. [17]

A soft set $\left(\zeta_{S_{1}}, \Lambda_{1}\right)$ is a soft subset of another soft set $\left(\zeta_{S_{2}}, \Lambda_{2}\right)$ if

(i) $\Lambda_{1} \subseteq \Lambda_{2}$, and

(ii) $\zeta_{S_{1}}(\omega) \subseteq \zeta_{S_{2}}(\omega)$ for all $\omega \in \Lambda_{1}$.

Definition 3. [17]

Union of two soft sets $\left(\zeta_{S_{1}}, \Lambda_{1}\right)$ and $\left(\zeta_{S_{2}}, \Lambda_{2}\right)$ is a soft set $\left(\zeta_{S_{3}}, \Lambda_{3}\right)$ with $\Lambda_{3}=\Lambda_{1} \cup \Lambda_{2}$ and for $\omega \in \Lambda_{3}$,

$$
\zeta_{S_{3}}(\omega)=\left\{\begin{array}{cc}
\zeta_{S_{1}}(\omega) & \omega \in\left(\Lambda_{1} \backslash \Lambda_{2}\right) \\
\zeta_{S_{2}}(\omega) & \omega \in\left(\Lambda_{2} \backslash \Lambda_{1}\right) \\
\zeta_{S_{1}}(\omega) \cup \zeta_{S_{2}}(\omega) & \omega \in\left(\Lambda_{1} \cap \Lambda_{2}\right)
\end{array}\right.
$$

Definition 4. [17]

Intersection of two soft sets $\left(\zeta_{S_{1}}, \Lambda_{1}\right)$ and $\left(\zeta_{S_{2}}, \Lambda_{2}\right)$ is a soft set $\left(\zeta_{S_{3}}, \Lambda_{3}\right)$ with $\Lambda_{3}=$ $\Lambda_{1} \cap \Lambda_{2}$ and for $\omega \in \Lambda_{3}$,

$$
\zeta_{S_{3}}(\omega)=\zeta_{S_{1}}(\omega) \cap \zeta_{S_{2}}(\omega)
$$

Definition 5. [17]

AND operation of two soft sets $\left(\zeta_{S_{1}}, \Lambda_{1}\right)$ and $\left(\zeta_{S_{2}}, \Lambda_{2}\right)$, denoted by $\left(\zeta_{S_{1}}, \Lambda_{1}\right) \wedge\left(\zeta_{S_{2}}, \Lambda_{2}\right)$, is a soft set $\left(\zeta_{S_{3}}, \Lambda_{3}\right)$ with $\Lambda_{3}=\Lambda_{1} \times \Lambda_{2}$ and for $\omega \in \Lambda_{3}$,

$$
\zeta_{S_{3}}(\omega)=\zeta_{S_{1}}(\omega) \cap \zeta_{S_{2}}(\omega)
$$

For more details on soft set, see [19, 17, 22, 2, 3, 4, 39, 6, 16]

Definition 6. [7]

A soft set $\left(\zeta_{S}, \Lambda\right)$ is said to be a bijective soft set, if

(i) $\cup_{\epsilon \in \Lambda} \zeta_{S}(\epsilon)=\mathcal{U}$

(ii)For any two parameters $\epsilon_{i}, \epsilon_{j} \in \Lambda, \epsilon_{i} \neq \epsilon_{j}, \zeta_{S}\left(\epsilon_{i}\right) \cap \zeta_{S}\left(\epsilon_{j}\right)=\emptyset$

\section{Bijective Hypersoft Set (BHS-SET)}

In this section, we start with definition of hypersoft set with example then we characterize the theory of bijective hypersoft set.

Definition 7. [40]

The pair $(\mathfrak{F}, \mathcal{G})$ is called a hypersoft set over $\mathfrak{U}$, where $\mathcal{G}$ is the cartesian product of $\mathrm{n}$ disjoint attribute-valued sets $\mathcal{G}_{1}, \mathcal{G}_{2}, \mathcal{G}_{3}, \ldots, \mathcal{G}_{n}$ corresponding to $n$ distinct attributes $g_{1}, g_{2}, g_{3}, \ldots, g_{n}$ respectively and $\mathfrak{F}: \mathcal{G} \rightarrow \mathcal{P}(\mathfrak{U})$. The collection of all hypersoft sets is denoted by $\Omega_{(\mathfrak{F}, \mathcal{G})}$. 
Example 3.1. Suppose that Mr. Smith wants to buy a mobile from a mobile market. There are sixteen kinds of mobiles (options) which form the set of discourse

$$
\mathfrak{U}=\left\{m_{1}, m_{2}, m_{3}, m_{4}, m_{5}, \ldots \ldots, m_{16}\right\}
$$

. The best selection may be evaluated by observing the attributes i.e. $g_{1}=$ Camera Resolution, $g_{2}=$ storage, and $g_{3}=$ Battery power. The attribute-valued sets corresponding to these attributes are:

$\mathcal{G}_{1}=\left\{g_{11}, g_{12}\right\}$

$\mathcal{G}_{2}=\left\{g_{21}, g_{22}\right\}$

$\mathcal{G}_{3}=\left\{g_{31}, g_{32}\right\}$

then $\mathcal{G}=\mathcal{G}_{1} \times \mathcal{G}_{2} \times \mathcal{G}_{3}$

$\mathcal{G}=\left\{\delta_{1}, \delta_{2}, \delta_{3}, \delta_{4}, \ldots ., \delta_{8}\right\}$ where each $\delta_{i}, i=1,2, \ldots, 8$, is a 3 -tuple element.

The hypersoft set $(\mathfrak{F}, \mathcal{G})$ is given as

$(\mathfrak{F}, \mathcal{G})=\left\{\begin{array}{l}\left(\delta_{1},\left\{m_{1}, m_{2}, m_{9}, m_{14}, m_{16}\right\}\right),\left(\delta_{2},\left\{m_{1}, m_{2}, m_{3}, m_{10}, m_{12}\right\}\right), \\ \left(\delta_{3},\left\{m_{2}, m_{3}, m_{4}, m_{9}, m_{13}, m_{14}\right\}\right),\left(\delta_{4},\left\{m_{4}, m_{5}, m_{6}, m_{10}, m_{15}, m_{16}\right\}\right), \\ \left(\delta_{5},\left\{m_{6}, m_{7}, m_{8}, m_{10}, m_{12}\right\}\right),\left(\delta_{6},\left\{m_{2}, m_{3}, m_{4}, m_{7}, m_{9}\right\}\right), \\ \left(\delta_{7},\left\{m_{1}, m_{3}, m_{5}, m_{6}, m_{8}, m_{10}, m_{12}, m_{14}, m_{16}\right\}\right), \\ \left(\delta_{8},\left\{m_{2}, m_{3}, m_{6}, m_{7}, m_{9}, m_{11}, m_{13}, m_{15}\right\}\right)\end{array}\right\}$ and tabular representation of $(\mathfrak{F}, \mathcal{G})$ is given in TABLE 1 where if $m_{i} \in \mathfrak{F}\left(\delta_{i}\right)$ then 1 otherwise 0 .

\begin{tabular}{|l|l|l|l|l|l|l|l|l|}
\hline$\ldots$. & $\mathfrak{F}\left(\delta_{1}\right)$ & $\mathfrak{F}\left(\delta_{2}\right)$ & $\mathfrak{F}\left(\delta_{3}\right)$ & $\mathfrak{F}\left(\delta_{4}\right)$ & $\mathfrak{F}\left(\delta_{5}\right)$ & $\mathfrak{F}\left(\delta_{6}\right)$ & $\mathfrak{F}\left(\delta_{7}\right)$ & $\mathfrak{F}\left(\delta_{8}\right)$ \\
\hline$m_{1}$ & 1 & 1 & 0 & 0 & 0 & 0 & 1 & 0 \\
\hline$m_{2}$ & 1 & 1 & 1 & 0 & 0 & 1 & 0 & 1 \\
\hline$m_{3}$ & 0 & 1 & 1 & 0 & 0 & 1 & 1 & 1 \\
\hline$m_{4}$ & 0 & 0 & 1 & 1 & 0 & 1 & 0 & 0 \\
\hline$m_{5}$ & 0 & 0 & 0 & 1 & 0 & 0 & 1 & 0 \\
\hline$m_{6}$ & 0 & 0 & 0 & 1 & 1 & 0 & 1 & 1 \\
\hline$m_{7}$ & 0 & 0 & 0 & 0 & 1 & 1 & 0 & 1 \\
\hline$m_{8}$ & 0 & 0 & 0 & 0 & 1 & 0 & 1 & 0 \\
\hline$m_{9}$ & 1 & 0 & 1 & 0 & 0 & 1 & 0 & 1 \\
\hline$m_{10}$ & 0 & 1 & 0 & 1 & 1 & 0 & 1 & 0 \\
\hline$m_{11}$ & 0 & 0 & 0 & 0 & 0 & 0 & 0 & 1 \\
\hline$m_{12}$ & 0 & 1 & 0 & 0 & 1 & 0 & 1 & 0 \\
\hline$m_{13}$ & 0 & 0 & 1 & 0 & 0 & 0 & 0 & 1 \\
\hline$m_{14}$ & 1 & 0 & 1 & 0 & 0 & 0 & 1 & 0 \\
\hline$m_{15}$ & 0 & 0 & 0 & 1 & 0 & 0 & 0 & 1 \\
\hline$m_{16}$ & 1 & 0 & 0 & 1 & 0 & 0 & 1 & 0 \\
\hline
\end{tabular}

TABLE 1. The table form of hypersoft $\operatorname{set}(\mathfrak{F}, \mathcal{G})$

Definition 8. A hypersoft set $(\mathfrak{F}, \mathcal{G})$ is said to be bijective hypersoft set if

(i) $\bigcup_{\delta \in \mathcal{G}} \mathfrak{F}(\delta)=\mathfrak{U}$ 
(ii) For any two $\delta_{i}, \delta_{j} \in \mathcal{G}, \delta_{i} \neq \delta_{j}, \mathfrak{F}\left(\delta_{i}\right) \cap \mathfrak{F}\left(\delta_{j}\right)=\emptyset$

Alternatively, a mapping $\mathfrak{F}: \mathcal{G} \rightarrow \mathcal{P}(\mathfrak{U})$ can be transformed to a bijective function $\mathfrak{F}$ : $\mathcal{G} \rightarrow \mathcal{P}_{1}$, where $\mathcal{P}_{1} \subseteq \mathcal{P}(\mathfrak{U})$ having disjoint sets $\mathfrak{F}\left(e_{i}\right)$ as elements. The collection of all bijective hypersoft sets over $\mathfrak{U}$ is denoted by $\Omega_{B H S S}$.

Example 3.2. Consider Example 3.1, we have bijective hypersoft set

$$
(\mathfrak{F}, \mathcal{G})=\left\{\begin{array}{l}
\left(\delta_{1},\left\{m_{1}, m_{2}\right\}\right),\left(\delta_{2},\left\{m_{3}, m_{10}\right\}\right),\left(\delta_{3},\left\{m_{4}, m_{14}\right\}\right),\left(\delta_{4},\left\{m_{5}, m_{15}\right\}\right), \\
\left(\delta_{5},\left\{m_{6}, m_{12}\right\}\right),\left(\delta_{6},\left\{m_{7}, m_{9}\right\}\right),\left(\delta_{7},\left\{m_{8}, m_{16}\right\}\right),\left(\delta_{8},\left\{m_{11}, m_{13}\right\}\right)
\end{array}\right\}
$$

and its tabular representation is given in TABLE 2.

\begin{tabular}{|l|l|l|l|l|l|l|l|l|}
\hline$\ldots$. & $\mathfrak{F}\left(\delta_{1}\right)$ & $\mathfrak{F}\left(\delta_{2}\right)$ & $\mathfrak{F}\left(\delta_{3}\right)$ & $\mathfrak{F}\left(\delta_{4}\right)$ & $\mathfrak{F}\left(\delta_{5}\right)$ & $\mathfrak{F}\left(\delta_{6}\right)$ & $\mathfrak{F}\left(\delta_{7}\right)$ & $\mathfrak{F}\left(\delta_{8}\right)$ \\
\hline$m_{1}$ & 1 & 0 & 0 & 0 & 0 & 0 & 0 & 0 \\
\hline$m_{2}$ & 1 & 0 & 0 & 0 & 0 & 0 & 0 & 0 \\
\hline$m_{3}$ & 0 & 1 & 0 & 0 & 0 & 0 & 0 & 0 \\
\hline$m_{4}$ & 0 & 0 & 1 & 0 & 0 & 0 & 0 & 0 \\
\hline$m_{5}$ & 0 & 0 & 0 & 1 & 0 & 0 & 0 & 0 \\
\hline$m_{6}$ & 0 & 0 & 0 & 0 & 1 & 0 & 0 & 0 \\
\hline$m_{7}$ & 0 & 0 & 0 & 0 & 0 & 1 & 0 & 0 \\
\hline$m_{8}$ & 0 & 0 & 0 & 0 & 0 & 0 & 1 & 0 \\
\hline$m_{9}$ & 0 & 0 & 0 & 0 & 0 & 1 & 0 & 0 \\
\hline$m_{10}$ & 0 & 1 & 0 & 0 & 0 & 0 & 0 & 0 \\
\hline$m_{11}$ & 0 & 0 & 0 & 0 & 0 & 0 & 0 & 1 \\
\hline$m_{12}$ & 0 & 0 & 0 & 0 & 1 & 0 & 0 & 0 \\
\hline$m_{13}$ & 0 & 0 & 0 & 0 & 0 & 0 & 0 & 1 \\
\hline$m_{14}$ & 0 & 0 & 1 & 0 & 0 & 0 & 0 & 0 \\
\hline$m_{15}$ & 0 & 0 & 0 & 1 & 0 & 0 & 0 & 0 \\
\hline$m_{16}$ & 0 & 0 & 0 & 0 & 0 & 0 & 1 & 0 \\
\hline
\end{tabular}

TABLE 2. The table form of bijective hypersoft set $(\mathfrak{F}, \mathcal{G})$

Definition 9. AND operation of two hypersoft sets $\left(\mathfrak{F}_{1}, \mathcal{G}_{1}\right)$ and $\left(\mathfrak{F}_{2}, \mathcal{G}_{2}\right)$, denoted by $\left(\mathfrak{F}_{1}, \mathcal{G}_{1}\right) \wedge\left(\mathfrak{F}_{2}, \mathcal{G}_{2}\right)$, is a hypersoft set $\left.\left(\mathfrak{F}_{3}, \mathcal{G}_{3}\right)\right)$ with $\mathcal{G}_{3}=\mathcal{G}_{1} \times \mathcal{G}_{2}$ and for $\delta \in \mathcal{G}_{3}$,

$$
\mathfrak{F}_{3}(\delta)=\mathfrak{F}_{1}(\delta) \cap \mathfrak{F}_{2}(\delta)
$$

Theorem 3.3. If $\left(\mathfrak{F}_{1}, \mathcal{H}_{1}\right),\left(\mathfrak{F}_{2}, \mathcal{H}_{2}\right) \in \Omega_{B H S S}$ then $\left(\mathfrak{F}_{1}, \mathcal{H}_{1}\right) \wedge\left(\mathfrak{F}_{2}, \mathcal{H}_{2}\right) \in \Omega_{B H S S}$

Proof. According to Definition 9, we have

$\left(\mathfrak{F}_{1}, \mathcal{H}_{1}\right) \wedge\left(\mathfrak{F}_{2}, \mathcal{H}_{2}\right)=\left(\mathfrak{F}_{3}, \mathcal{H}_{3}\right)$, where $\mathcal{H}_{3}=\mathcal{H}_{1} \times \mathcal{H}_{2}$ and $\mathfrak{F}_{3}\left(h_{1}, h_{2}\right)=\mathfrak{F}_{1}\left(h_{1}\right) \cap$ $\mathfrak{F}_{2}\left(h_{2}\right), \forall\left(h_{1}, h_{2}\right) \in \mathcal{H}_{3}$. Consider $\varepsilon \in \mathcal{H}_{3}$ is a parameter of $\left(\mathfrak{F}_{3}, \mathcal{H}_{3}\right)$ then

$$
\mathfrak{F}_{3}(\varepsilon)=\mathfrak{F}_{1}\left(h_{1}\right) \cap \mathfrak{F}_{2}\left(h_{2}\right)
$$

$\therefore \bigcup_{\varepsilon \in \mathcal{H}_{2}} \mathfrak{F}_{3}(\varepsilon)=\bigcup_{h_{1} \in \mathcal{H}_{1}} \bigcup_{h_{2} \in \mathcal{H}_{2}} \mathfrak{F}_{1}\left(h_{1}\right) \cap \mathfrak{F}_{2}\left(h_{2}\right)=\bigcup_{h_{1} \in \mathcal{H}_{1}} \mathfrak{F}_{1}\left(h_{1}\right) \cap\left(\bigcup_{h_{2} \in \mathcal{H}_{2}} \mathfrak{F}_{2}\left(h_{2}\right)\right)=$ $\bigcup_{h_{1} \in \mathcal{H}_{1}} \mathfrak{F}_{1}\left(h_{1}\right) \bigcap \mathfrak{U}=\mathfrak{U}$. Let $\varepsilon_{i}, \varepsilon_{j} \in \mathcal{H}_{3}, \varepsilon_{i} \neq \varepsilon_{j}, \varepsilon_{i}=\alpha_{1} \times \beta_{1}, \alpha_{1} \in \mathcal{H}_{1}, \beta_{1} \in \mathcal{H}_{2}, \varepsilon_{j}=$ 
$\alpha_{2} \times \beta_{2}, \alpha_{2} \in \mathcal{H}_{1}, \beta_{2} \in \mathcal{H}_{2}$. Then

$$
\mathfrak{F}_{3}\left(\varepsilon_{i}\right) \bigcap \mathfrak{F}_{3}\left(\varepsilon_{j}\right)=\left(\mathfrak{F}_{1}\left(\alpha_{1}\right) \bigcap \mathfrak{F}_{2}\left(\beta_{1}\right)\right) \bigcap\left(\mathfrak{F}_{1}\left(\alpha_{2}\right) \bigcap \mathfrak{F}_{2}\left(\beta_{2}\right)\right)=\emptyset .
$$

Hence $\left(\mathfrak{F}_{3}, \mathcal{H}_{3}\right)=\left(\mathfrak{F}_{1}, \mathcal{H}_{1}\right) \wedge\left(\mathfrak{F}_{2}, \mathcal{H}_{2}\right)$ is a bijective hypersoft set.

Definition 10. A hypersoft set $(\mathfrak{F}, \mathcal{G})$ is called a null hypersoft set, denoted by $(\mathfrak{F}, \mathcal{G})_{\Phi}$, if $\mathfrak{F}(\delta)=\emptyset$, for all $\delta \in \mathcal{G}$.

Definition 11. Union of two hypersoft sets $\left(\mathfrak{F}_{1}, \mathcal{G}_{1}\right)$ and $\left(\mathfrak{F}_{2}, \mathcal{G}_{2}\right)$, denoted by $\left(\mathfrak{F}_{1}, \mathcal{G}_{1}\right) \cup$ $\left(\mathfrak{F}_{2}, \mathcal{G}_{2}\right)$, is a hypersoft set $\left(\mathfrak{F}_{3}, \mathcal{G}_{3}\right)$ with $\mathcal{G}_{3}=\mathcal{G}_{1} \cup \mathcal{G}_{2}$ and for $\delta \in \mathcal{G}_{3}$,

$$
\mathfrak{F}_{3}(\delta)=\left\{\begin{array}{cc}
\mathfrak{F}_{1}(\delta) & \delta \in\left(\mathcal{G}_{1} \backslash \mathcal{G}_{2}\right) \\
\mathfrak{F}_{2}(\delta) & \delta \in\left(\mathcal{G}_{2} \backslash \mathcal{G}_{1}\right) \\
\mathfrak{F}_{1}(\delta) \cup \mathfrak{F}_{2}(\delta) & \delta \in\left(\mathcal{G}_{1} \cap \mathcal{G}_{2}\right)
\end{array}\right.
$$

Theorem 3.4. If $(\mathfrak{F}, \mathcal{H}) \in \Omega_{B H S S}$ then $(\mathfrak{F}, \mathcal{H}) \cup(\mathfrak{F}, \mathcal{H})_{\Phi} \in \Omega_{B H S S}$.

Proof. Let $(\mathfrak{F}, \mathcal{H})_{\Phi}=\left(\mathfrak{F}_{\Phi}, \mathcal{H}_{1}\right)$, then from Definition 10 and Definition 11, we have $\left(\mathfrak{F}_{2}, \mathcal{H}_{2}\right)=(\mathfrak{F}, \mathcal{H}) \cup\left(\mathfrak{F}_{\Phi}, \mathcal{H}_{1}\right)$

$$
=\left\{\begin{array}{ccc}
\mathfrak{F}(\epsilon) & ; \epsilon \in \mathcal{H}-\mathcal{H}_{1} \\
\mathfrak{F}_{\Phi}(\epsilon)=\emptyset & ; \epsilon \in \mathcal{H}_{1}-\mathcal{H}=\left(\mathfrak{F}, \mathcal{H} \cup \mathcal{H}_{1}\right) \\
\mathfrak{F}(\epsilon) \cup \mathfrak{F}_{\Phi}(\epsilon)=\mathfrak{F}(\epsilon) \cup \emptyset & & ; \epsilon \in \mathfrak{F} \cap \mathfrak{F}_{1}
\end{array}\right.
$$

where $\epsilon \in \mathcal{H}_{2}$ and $\left(\mathfrak{F}, \mathcal{H}_{1}\right) \subset\left(\mathfrak{F}, \mathcal{H} \cup \mathcal{H}_{1}\right)$ is a Null hypersoft set, implies $\left(\mathfrak{F}_{2}, \mathcal{H}_{2}\right)=\left(\mathfrak{F}, \mathcal{H} \cup \mathcal{H}_{1}\right)$ is a bijective hypersoft set over $\mathfrak{U}$.

Definition 12. Let $\mathfrak{U}_{1} \subset \mathfrak{U}$ and $(\mathfrak{F}, \mathcal{H}) \in \Omega_{B H S S}$. The operation of $(\mathfrak{F}, \mathcal{H})$ restricted AND $\mathfrak{U}_{1}$, denoted by $(\mathfrak{F}, \mathcal{H}) \bigwedge_{\mathcal{R}} \mathfrak{U}_{1}$, is defined as

$$
\bigcup_{\delta \in \mathcal{H}}\left\{\mathfrak{F}(\delta): \mathfrak{F}(\delta) \subseteq \mathfrak{U}_{1}\right\} .
$$

Example 3.5. Let $\mathfrak{U}=\left\{u_{1}, u_{2}, u_{3}, \ldots ., u_{8}\right\}$ and $\mathfrak{U}_{1}=\left\{u_{1}, u_{2}, u_{3}, u_{4}\right\}$. If $(\mathfrak{F}, \mathcal{H}) \in$ $\Omega_{B H S S}$ with

$$
(\mathfrak{F}, \mathcal{H})=\left\{\left(\delta_{1},\left\{u_{1}, u_{2}\right\}\right),\left(\delta_{2},\left\{u_{3}, u_{4}\right\}\right),\left(\delta_{3},\left\{u_{5}, u_{6}\right\}\right),\left(\delta_{4},\left\{u_{7}, u_{8}\right\}\right)\right\}
$$

then

$$
(\mathfrak{F}, \mathcal{H}) \bigwedge_{\mathcal{R}} \mathfrak{U}_{1}=\left\{u_{1}, u_{2}\right\} \cup\left\{u_{3}, u_{4}\right\}=\left\{u_{1}, u_{2}, u_{3}, u_{4}\right\}
$$

Definition 13. Let $\mathfrak{U}_{1} \subset \mathfrak{U}$ and $(\mathfrak{F}, \mathcal{H}) \in \Omega_{B H S S}$. The operation of $(\mathfrak{F}, \mathcal{H})$ relaxed AND $\mathfrak{U}_{1}$, denoted by $(\mathfrak{F}, \mathcal{H}) \bigwedge_{\mathcal{R e l}} \mathfrak{U}_{1}$, is defined as

$$
\bigcup_{\delta \in \mathcal{H}}\left\{\mathfrak{F}(\delta): \mathfrak{F}(\delta) \cap \mathfrak{U}_{1} \neq \emptyset\right\} .
$$

Example 3.6. Let $\mathfrak{U}=\left\{u_{1}, u_{2}, u_{3}, \ldots, u_{8}\right\}$ and $\mathfrak{U}_{1}=\left\{u_{1}, u_{2}, u_{3}, u_{4}\right\}$. If $(\mathfrak{F}, \mathcal{H}) \in$ $\Omega_{B H S S}$ with

$$
(\mathfrak{F}, \mathcal{H})=\left\{\left(\delta_{1},\left\{u_{1}, u_{8}\right\}\right),\left(\delta_{2},\left\{u_{3}, u_{7}\right\}\right),\left(\delta_{3},\left\{u_{2}, u_{6}\right\}\right),\left(\delta_{4},\left\{u_{4}, u_{5}\right\}\right)\right\}
$$


then

$(\mathfrak{F}, \mathcal{H}) \bigwedge_{\mathcal{R} e l} \mathfrak{U}_{1}=\left\{u_{1}, u_{8}\right\} \cup\left\{u_{3}, u_{7}\right\} \cup\left\{u_{2}, u_{6}\right\} \cup\left\{u_{4}, u_{5}\right\}=\left\{u_{1}, u_{2}, u_{3}, u_{4}, u_{5}, u_{6}, u_{7}, u_{8}\right\}=\mathfrak{U}$

Definition 14. If $(\mathfrak{F}, \mathcal{H}) \in \Omega_{B H S S}$ then its boundary region with respect to $\mathfrak{U}_{1} \subset \mathfrak{U}$, denoted by $(\mathfrak{F}, \mathcal{H})_{\bullet}$, is defined as

$$
(\mathfrak{F}, \mathcal{H}) \bullet=\left((\mathfrak{F}, \mathcal{H}) \bigwedge_{\mathcal{R} e l} \mathfrak{U}_{1}\right) \backslash\left((\mathfrak{F}, \mathcal{H}) \bigwedge_{\mathcal{R}} \mathfrak{U}_{1}\right)
$$

Example 3.7. Consider $\mathfrak{U}$ and $\mathfrak{U}_{1}$ as in Example 3.6, we have

$$
(\mathfrak{F}, \mathcal{H})=\left\{\left(\delta_{1},\left\{u_{1}\right\}\right),\left(\delta_{2},\left\{u_{3}, u_{4}\right\}\right),\left(\delta_{3},\left\{u_{2}, u_{6}\right\}\right),\left(\delta_{4},\left\{u_{7}, u_{5}\right\}\right)\right\}
$$

. Now

$$
(\mathfrak{F}, \mathcal{H}) \bigwedge_{\mathcal{R} e l} \mathfrak{U}_{1}=\left\{u_{1}\right\} \cup\left\{u_{3}, u_{4}\right\} \cup\left\{u_{2}, u_{6}\right\}=\left\{u_{1}, u_{2}, u_{3}, u_{4}, u_{6}\right\}
$$

and

$$
(\mathfrak{F}, \mathcal{H}) \bigwedge_{\mathcal{R}} \mathfrak{U}_{1}=\left\{u_{1}\right\} \cup\left\{u_{3}, u_{4}\right\}=\left\{u_{1}, u_{3}, u_{4}\right\}
$$

therefore

$$
(\mathfrak{F}, \mathcal{H}) \bullet=\left\{u_{2}, u_{6}\right\}
$$

Definition 15. If $\left(\mathfrak{F}_{1}, \mathcal{H}_{1}\right),\left(\mathfrak{F}_{2}, \mathcal{H}_{2}\right) \in \Omega_{B H S S}$ with $\mathcal{H}_{1} \cap \mathcal{H}_{2}=\emptyset$ then $\left(\mathfrak{F}_{1}, \mathcal{H}_{1}\right)$ is said to depend on $\left(\mathfrak{F}_{2}, \mathcal{H}_{2}\right)$ to a degree $\kappa \in[0,1]$, denoted by $\left(\mathfrak{F}_{1}, \mathcal{H}_{1}\right) \underset{\kappa}{\Rightarrow}\left(\mathfrak{F}_{2}, \mathcal{H}_{2}\right)$, if

$$
\kappa=\Gamma\left(\left(\mathfrak{F}_{1}, \mathcal{H}_{1}\right),\left(\mathfrak{F}_{2}, \mathcal{H}_{2}\right)\right)=\frac{\left|\bigcup_{\delta \in \mathcal{H}_{2}}\left\{\left(\mathfrak{F}_{1}, \mathcal{H}_{1}\right) \bigwedge_{\mathcal{R}} \mathfrak{F}_{2}(\delta)\right\}\right|}{|\mathfrak{U}|}
$$

where $|\cdot|$ = cardinality of a set.

Note:

(i) If $\kappa=1$ then $\left(\mathfrak{F}_{1}, \mathcal{H}_{1}\right)$ is full depended on $\left(\mathfrak{F}_{2}, \mathcal{H}_{2}\right)$.

(ii) If $\kappa=0$ then $\left(\mathfrak{F}_{1}, \mathcal{H}_{1}\right)$ is not depended on $\left(\mathfrak{F}_{2}, \mathcal{H}_{2}\right)$.

Example 3.8. Consider $\mathfrak{U}$ as in Example 3.6, we have

$$
\left(\mathfrak{F}_{1}, \mathcal{H}_{1}\right)=\left\{\begin{array}{c}
\left(\delta_{1},\left\{u_{1}\right\}\right),\left(\delta_{2},\left\{u_{3}\right\}\right),\left(\delta_{3},\left\{u_{6}\right\}\right),\left(\delta_{4},\left\{u_{7}\right\}\right), \\
\left(\delta_{5},\left\{u_{5}\right\}\right),\left(\delta_{6},\left\{u_{8}\right\}\right),\left(\delta_{7},\left\{u_{2}\right\}\right),\left(\delta_{8},\left\{u_{4}\right\}\right)
\end{array}\right\}
$$

and

$$
\left(\mathfrak{F}_{2}, \mathcal{H}_{2}\right)=\left\{\left(\delta_{9},\left\{u_{1}, u_{2}\right\}\right),\left(\delta_{10},\left\{u_{3}, u_{4}\right\}\right),\left(\delta_{11},\left\{u_{5}\right\}\right),\left(\delta_{12},\left\{u_{7}, u_{8}\right\}\right)\right\}
$$

Now

$$
\begin{aligned}
& \left(\mathfrak{F}_{1}, \mathcal{H}_{1}\right) \bigwedge_{\mathcal{R}} \mathfrak{F}_{2}\left(\delta_{9}\right)=\left\{u_{1}\right\} \cup\left\{u_{2}\right\}=\left\{u_{1}, u_{2}\right\} \\
& \left(\mathfrak{F}_{1}, \mathcal{H}_{1}\right) \bigwedge_{\mathcal{R}} \mathfrak{F}_{2}\left(\delta_{10}\right)=\left\{u_{3}\right\} \cup\left\{u_{4}\right\}=\left\{u_{3}, u_{4}\right\}
\end{aligned}
$$




$$
\begin{gathered}
\left(\mathfrak{F}_{1}, \mathcal{H}_{1}\right) \bigwedge_{\mathcal{R}} \mathfrak{F}_{2}\left(\delta_{11}\right)=\left\{u_{5}\right\} \\
\left(\mathfrak{F}_{1}, \mathcal{H}_{1}\right) \bigwedge_{\mathcal{R}} \mathfrak{F}_{2}\left(\delta_{12}\right)=\left\{u_{7}\right\} \cup\left\{u_{8}\right\}=\left\{u_{7}, u_{8}\right\}
\end{gathered}
$$

therefore

$$
\bigcup_{\delta \in \mathcal{H}_{2}}\left\{\left(\mathfrak{F}_{1}, \mathcal{H}_{1}\right) \bigwedge_{\mathcal{R}} \mathfrak{F}_{2}(\delta)\right\}=\left\{u_{1}, u_{2}, u_{3}, u_{4}, u_{5}, u_{7}, u_{8}\right\}
$$

with

$$
\kappa=\frac{7}{8}=0.875
$$

Definition 16. Let $(\mathfrak{F}, \mathcal{H}),(\mathfrak{L}, \mathcal{G}) \in \Omega_{B H S S}$. The triple $((\mathfrak{F}, \mathcal{H}),(\mathfrak{L}, \mathcal{G}), \mathfrak{U})$ is said to form a bijective hypersoft decision system over $\mathfrak{U}$, denoted by $\mathbb{D}_{B H}$, if

(i) there exists a condition hypersoft set $(\mathfrak{F}, \mathcal{H})=\bigcup_{i=1}^{n}\left(\mathfrak{F}_{i}, \mathcal{H}_{i}\right)$ for all $\left(\mathfrak{F}_{i}, \mathcal{H}_{i}\right) \in$ $\Omega_{B H S S}$

with $\mathcal{H}_{i} \cap \mathcal{H}_{j}=\emptyset, \quad i \neq j$

(ii) there exists a decision hypersoft set $(\mathfrak{L}, \mathcal{G})$ for which $\mathcal{G} \cap \mathcal{H}_{i}=\emptyset$.

Example 3.9. Consider $\mathfrak{U}$ as in Example 3.6, we have

$$
\begin{gathered}
\left(\mathfrak{F}_{1}, \mathcal{H}_{1}\right)=\left\{\left(\delta_{1},\left\{u_{1}\right\}\right),\left(\delta_{2},\left\{u_{2}\right\}\right),\left(\delta_{3},\left\{u_{3}\right\}\right)\right\} \\
\left(\mathfrak{F}_{2}, \mathcal{H}_{2}\right)=\left\{\left(\delta_{4},\left\{u_{1}, u_{2}\right\}\right),\left(\delta_{5},\left\{u_{3}, u_{4}\right\}\right),\left(\delta_{6},\left\{u_{5}, u_{6}\right\}\right)\right\} \\
\left(\mathfrak{F}_{3}, \mathcal{H}_{3}\right)=\left\{\left(\delta_{7},\left\{u_{1}, u_{2}, u_{3}\right\}\right),\left(\delta_{8},\left\{u_{4}, u_{5}, u_{6}\right\}\right),\left(\delta_{9},\left\{u_{7}, u_{8}\right\}\right)\right\}
\end{gathered}
$$

and

$$
(\mathfrak{L}, \mathcal{G})=\left\{\left(\delta_{10},\left\{u_{1}, u_{3}, u_{5}\right\}\right),\left(\delta_{11},\left\{u_{2}, u_{4}, u_{8}\right\}\right),\left(\delta_{12},\left\{u_{6}, u_{7}\right\}\right)\right\}
$$

therefore

$$
\mathbb{D}_{B H}=\left(\bigcup_{i=1}^{3}\left(\mathfrak{F}_{i}, \mathcal{H}_{i}\right),(\mathfrak{L}, \mathcal{G}), \mathfrak{U}\right)
$$

Definition 17. The bijective hypersoft dependency between $\left(\mathfrak{F}_{1}, \mathcal{H}_{1}\right) \wedge\left(\mathfrak{F}_{2}, \mathcal{H}_{2}\right) \wedge \ldots \wedge\left(\mathfrak{F}_{n}, \mathcal{H}_{n}\right)$ and $(\mathfrak{L}, \mathcal{G})$ is called bijective hypersoft decision system dependency of $\mathbb{D}_{B H}$ and defined by $\kappa=\Gamma\left(\bigwedge_{i=1}^{n}\left(\mathfrak{F}_{i}, \mathcal{H}_{i}\right),(\mathfrak{L}, \mathcal{G})\right)$.

Example 3.10. Considering the $\mathfrak{U}$ from Example 3.6, Let we have

$$
\begin{gathered}
\left(\mathfrak{F}_{1}, \mathcal{H}_{1}\right)=\left\{\left(\delta_{1},\left\{u_{1}, u_{4}, u_{6}\right\}\right),\left(\delta_{2},\left\{u_{2}, u_{5}, u_{7}\right\}\right)\right\} \\
\left(\mathfrak{F}_{2}, \mathcal{H}_{2}\right)=\left\{\left(\delta_{3},\left\{u_{1}, u_{5}, u_{6}\right\}\right),\left(\delta_{4},\left\{u_{4}, u_{7}, u_{8}\right\}\right)\right\} \\
\left(\mathfrak{F}_{3}, \mathcal{H}_{3}\right)=\left\{\left(\delta_{5},\left\{u_{1}, u_{2}, u_{4}\right\}\right),\left(\delta_{6},\left\{u_{3}, u_{5}, u_{6}\right\}\right),\left(\delta_{7},\left\{u_{7}, u_{8}\right\}\right)\right\} \\
(\mathfrak{L}, \mathcal{G})=\left\{\left(\delta_{8},\left\{u_{1}, u_{4}, u_{5}, u_{6}\right\}\right),\left(\delta_{9},\left\{u_{2}, u_{3}, u_{7}, u_{8}\right\}\right)\right\}
\end{gathered}
$$


then

$(\mathfrak{F}, \mathcal{H})=\left(\mathfrak{F}_{1}, \mathcal{H}_{1}\right) \bigwedge\left(\mathfrak{F}_{2}, \mathcal{H}_{2}\right) \bigwedge\left(\mathfrak{F}_{3}, \mathcal{H}_{3}\right)=$

$$
\left\{\begin{array}{l}
\left(\varepsilon_{1}=\left(\delta_{1}, \delta_{3}, \delta_{5}\right),\left\{u_{1}\right\}\right),\left(\varepsilon_{2}=\left(\delta_{1}, \delta_{3}, \delta_{6}\right),\left\{u_{6}\right\}\right),\left(\varepsilon_{3}=\left(\delta_{1}, \delta_{3}, \delta_{7}\right), \emptyset\right) \\
\left(\varepsilon_{4}=\left(\delta_{1}, \delta_{4}, \delta_{5}\right),\left\{u_{4}\right\}\right),\left(\varepsilon_{5}=\left(\delta_{1}, \delta_{4}, \delta_{6}\right), \emptyset\right),\left(\varepsilon_{6}=\left(\delta_{1}, \delta_{4}, \delta_{7}\right), \emptyset\right) \\
\left(\varepsilon_{7}=\left(\delta_{2}, \delta_{3}, \delta_{5}\right), \emptyset\right),\left(\varepsilon_{8}=\left(\delta_{2}, \delta_{3}, \delta_{6}\right),\left\{u_{5}\right\}\right),\left(\varepsilon_{9}=\left(\delta_{2}, \delta_{3}, \delta_{7}\right), \emptyset\right) \\
\left(\varepsilon_{10}=\left(\delta_{2}, \delta_{4}, \delta_{5}\right), \emptyset\right),\left(\varepsilon_{11}=\left(\delta_{2}, \delta_{4}, \delta_{6}\right), \emptyset\right),\left(\varepsilon_{12}=\left(\delta_{2}, \delta_{4}, \delta_{7}\right),\left\{u_{7}\right\}\right)
\end{array}\right\} .
$$

The tabular representation of $\left(\mathfrak{F}_{1}, \mathcal{H}_{1}\right) \bigwedge\left(\mathfrak{F}_{2}, \mathcal{H}_{2}\right) \wedge\left(\mathfrak{F}_{3}, \mathcal{H}_{3}\right)$ is depicted in TABLE 3.

Now

$\left(\bigwedge_{i=1}^{3}\left(\mathfrak{F}_{i}, \mathcal{H}_{i}\right) \bigwedge_{\mathcal{R}}(\mathfrak{L}, \mathcal{G})\right)=\left\{u_{1}, u_{4}, u_{5}, u_{6}, u_{7}\right\}$

therefore

$\kappa=\Gamma\left(\bigwedge_{i=1}^{3}\left(\mathfrak{F}_{i}, \mathcal{H}_{i}\right),(\mathfrak{L}, \mathcal{G})\right)=\frac{5}{8}=0.625$

\begin{tabular}{|l|l|l|l|l|l|l|l|l|}
\hline$\ldots$ & $u_{1}$ & $u_{2}$ & $u_{3}$ & $u_{4}$ & $u_{5}$ & $u_{6}$ & $u_{7}$ & $u_{8}$ \\
\hline $\mathfrak{F}\left(\varepsilon_{1}\right)$ & 1 & 0 & 0 & 0 & 0 & 0 & 0 & 0 \\
\hline $\mathfrak{F}\left(\varepsilon_{2}\right)$ & 0 & 0 & 0 & 0 & 0 & 1 & 0 & 0 \\
\hline $\mathfrak{F}\left(\varepsilon_{3}\right)$ & 0 & 0 & 0 & 0 & 0 & 0 & 0 & 0 \\
\hline $\mathfrak{F}\left(\varepsilon_{4}\right)$ & 0 & 0 & 0 & 1 & 0 & 0 & 0 & 0 \\
\hline $\mathfrak{F}\left(\varepsilon_{5}\right)$ & 0 & 0 & 0 & 0 & 0 & 0 & 0 & 0 \\
\hline $\mathfrak{F}\left(\varepsilon_{6}\right)$ & 0 & 0 & 0 & 0 & 0 & 0 & 0 & 0 \\
\hline $\mathfrak{F}\left(\varepsilon_{7}\right)$ & 0 & 0 & 0 & 0 & 0 & 0 & 0 & 0 \\
\hline $\mathfrak{F}\left(\varepsilon_{8}\right)$ & 0 & 0 & 0 & 0 & 1 & 0 & 0 & 0 \\
\hline $\mathfrak{F}\left(\varepsilon_{9}\right)$ & 0 & 0 & 0 & 0 & 0 & 0 & 0 & 0 \\
\hline $\mathfrak{F}\left(\varepsilon_{10}\right)$ & 0 & 0 & 0 & 0 & 0 & 0 & 0 & 0 \\
\hline $\mathfrak{F}\left(\varepsilon_{11}\right)$ & 0 & 0 & 0 & 0 & 0 & 0 & 0 & 0 \\
\hline $\mathfrak{F}\left(\varepsilon_{12}\right)$ & 0 & 0 & 0 & 0 & 0 & 0 & 1 & 0 \\
\hline
\end{tabular}

TABLE 3. The table form of $\left(\mathfrak{F}_{1}, \mathcal{H}_{1}\right) \wedge\left(\mathfrak{F}_{2}, \mathcal{H}_{2}\right) \wedge\left(\mathfrak{F}_{3}, \mathcal{H}_{3}\right)$

Theorem 3.11. Let $\mathbb{D}_{B H}=((\mathfrak{F}, \mathcal{H}),(\mathfrak{L}, \mathcal{G}), \mathfrak{U})$, where $(\mathfrak{F}, \mathcal{H})=\bigcup_{i=1}^{n}\left(\mathfrak{F}_{i}, \mathcal{H}_{i}\right)$ and $\left(\mathfrak{F}_{i}, \mathcal{H}_{i}\right) \in$ $\Omega_{B H S S}$. If $\kappa=\gamma\left(\bigwedge_{i=1}^{n}\left(\mathfrak{F}_{i}, \mathcal{H}_{i}\right),(\mathfrak{L}, \mathcal{G})\right)$ and $\kappa_{1}=\gamma\left(\bigwedge_{i=1}^{m}\left(\mathfrak{F}_{i}, \mathcal{H}_{i}\right),(\mathfrak{L}, \mathcal{G})\right)$ with $m \leq n$ then $\kappa_{1} \leq \kappa$.

Proof. Suppose that $(\mathfrak{P}, \mathcal{C})=\bigwedge_{i=1}^{n}\left(\mathfrak{F}_{i}, \mathcal{H}_{i}\right),(\mathfrak{J}, \mathcal{K})=\bigwedge_{i=1}^{m}\left(\mathfrak{F}_{i}, \mathcal{H}_{i}\right)$ then we have

$$
\begin{aligned}
& \kappa=\gamma\left(\bigwedge_{i=1}^{n}\left(\mathfrak{F}_{i}, \mathcal{H}_{i}\right),(\mathfrak{L}, \mathcal{G})\right)=\frac{\left|\cup_{\varepsilon \in \mathcal{G}}(\mathfrak{P}, \mathcal{C}) \bigwedge_{\mathcal{R}} \mathfrak{L}(\varepsilon)\right|}{|\mathfrak{U}|}=\frac{\left|\cup_{\varepsilon \in \mathcal{G}}^{\cup} \underset{\alpha \in \mathcal{C}}{\cup}\{\mathfrak{P}(\alpha): \mathfrak{P}(\alpha) \subseteq \mathfrak{L}(\varepsilon)\}\right|}{|\mathfrak{U}|} \\
& \kappa_{1}=\gamma\left(\bigwedge_{i=1}^{m}\left(\mathfrak{F}_{i}, \mathcal{H}_{i}\right),(\mathfrak{L}, \mathcal{G})\right)=\frac{\left|\cup_{\varepsilon \in \mathcal{G}}(\mathfrak{J}, \mathcal{K}) \bigwedge_{\mathcal{R}} \mathfrak{L}(\varepsilon)\right|}{|\mathfrak{U}|}=\frac{\left|\cup_{\varepsilon \in \mathcal{G}} \cup_{\alpha \in \mathcal{K}}\{\mathfrak{J}(\alpha): \mathfrak{J}(\alpha) \subseteq \mathfrak{L}(\varepsilon)\}\right|}{|\mathfrak{U}|} .
\end{aligned}
$$


From Definition 2.6,

$\mathfrak{P}\left(\varepsilon_{1}, \varepsilon_{2}, . ., \varepsilon_{n}\right)=\mathfrak{F}_{1}\left(\varepsilon_{1}\right) \cap \mathfrak{F}_{2}\left(\varepsilon_{2}\right) \cap \cdot \cap \mathfrak{F}_{m}\left(\varepsilon_{m}\right) \cap \cdot \cap \mathfrak{F}_{n}\left(\varepsilon_{n}\right), \forall\left(\varepsilon_{1}, \varepsilon_{2}, . ., \varepsilon_{n}\right) \in \mathcal{H}_{1} \times \mathcal{H}_{2} \times \cdots \times \mathcal{H}_{n}$ $\mathfrak{J}\left(\varepsilon_{1}, \varepsilon_{2}, . ., \varepsilon_{m}\right)=\mathfrak{F}_{1}\left(\varepsilon_{1}\right) \cap \mathfrak{F}_{2}\left(\varepsilon_{2}\right) \cap . \cap \cap \mathfrak{F}_{m}\left(\varepsilon_{m}\right), \forall\left(\varepsilon_{1}, \varepsilon_{2}, . ., \varepsilon_{m}\right) \in \mathcal{H}_{1} \times \mathcal{H}_{2} \times \cdots \times \mathcal{H}_{m}$ $n>m$

$$
\mathfrak{P}\left(\varepsilon_{1}, \varepsilon_{2}, . ., \varepsilon_{n}\right) \supseteq \mathfrak{J}\left(\varepsilon_{1}, \varepsilon_{2}, . ., \varepsilon_{m}\right)
$$

and

$$
\cup_{\varepsilon \in \mathcal{C}} \mathfrak{P}(\varepsilon)=\mathfrak{U}, \underset{\varepsilon \in \mathcal{K}}{\cup} \mathfrak{J}(\varepsilon)=\mathfrak{U}
$$

Therefore,

$$
\begin{gathered}
\left|\cup_{\varepsilon \in \mathcal{C}}\{\mathfrak{P}(\varepsilon): \mathfrak{P}(\varepsilon) \subseteq \mathcal{G}(\varepsilon)\}\right| \geq\left|\cup_{\varepsilon \in \mathcal{K}}\{\mathfrak{J}(\varepsilon): \mathfrak{J}(\varepsilon) \subseteq \mathcal{G}(\varepsilon)\}\right| \\
\gamma\left(\bigwedge_{i=1}^{m}\left(\mathfrak{F}_{i}, \mathcal{H}_{i}\right),(\mathfrak{L}, \mathcal{G})\right) \leq \kappa
\end{gathered}
$$

Definition 18. Let $\mathbb{D}_{B H}=((\mathfrak{F}, \mathcal{H}),(\mathfrak{L}, \mathcal{G}), \mathfrak{U})$, where $(\mathfrak{F}, \mathcal{H})=\cup_{i=1}^{n}\left(\mathfrak{F}_{i}, \mathcal{H}_{i}\right)$ and $\bigcup_{i=1}^{m}\left(\mathfrak{F}_{i}, \mathcal{H}_{i}\right) \subset$ $(\mathfrak{F}, \mathcal{H})$. If $\gamma\left(\bigwedge_{i=1}^{n}\left(\mathfrak{F}_{i}, \mathcal{H}_{i}\right),(\mathfrak{L}, \mathcal{G})\right)=\gamma\left(\bigwedge_{i=1}^{m}\left(\mathfrak{F}_{i}, \mathcal{H}_{i}\right),(\mathfrak{L}, \mathcal{G})\right)=\kappa$ then $\bigcup_{i=1}^{m}\left(\mathfrak{F}_{i}, \mathcal{H}_{i}\right)$ is called a reduct of $\mathbb{D}_{B H}$.

Example 3.12. Considering the $\mathfrak{U}$ from Example 3.6 and sets from Example 3.10, let we have

$$
\begin{gathered}
\left(\mathfrak{F}_{1}, \mathcal{H}_{1}\right)=\left\{\left(\delta_{1},\left\{u_{1}, u_{4}, u_{6}\right\}\right),\left(\delta_{2},\left\{u_{2}, u_{5}, u_{7}\right\}\right)\right\} \\
\left(\mathfrak{F}_{2}, \mathcal{H}_{2}\right)=\left\{\left(\delta_{3},\left\{u_{1}, u_{5}, u_{6}\right\}\right),\left(\delta_{4},\left\{u_{4}, u_{7}, u_{8}\right\}\right)\right\} \\
\left(\mathfrak{F}_{3}, \mathcal{H}_{3}\right)=\left\{\left(\delta_{5},\left\{u_{1}, u_{2}, u_{4}\right\}\right),\left(\delta_{6},\left\{u_{3}, u_{5}, u_{6}\right\}\right),\left(\delta_{7},\left\{u_{7}, u_{8}\right\}\right)\right\} \\
(\mathfrak{L}, \mathcal{G})=\left\{\left(\delta_{8},\left\{u_{1}, u_{4}, u_{5}, u_{6}\right\}\right),\left(\delta_{9},\left\{u_{2}, u_{3}, u_{7}, u_{8}\right\}\right)\right\}
\end{gathered}
$$

then

$$
\left(\mathfrak{F}_{1}, \mathcal{H}_{1}\right) \bigwedge\left(\mathfrak{F}_{2}, \mathcal{H}_{2}\right)=\left\{\begin{array}{l}
\left(\varepsilon_{1}=\left(\delta_{1}, \delta_{3}\right),\left\{u_{1}, u_{6}\right\}\right),\left(\varepsilon_{2}=\left(\delta_{1}, \delta_{4}\right),\left\{u_{4}\right\}\right) \\
\left.\left(\varepsilon_{3}=\left(\delta_{2}, \delta_{3}\right),\left\{u_{5}\right\}\right)\left(\varepsilon_{4}=\left(\delta_{2}, \delta_{4}\right),\left\{u_{7}\right\}\right)\right)
\end{array}\right\}
$$

$\left(\bigwedge_{i=1}^{2}\left(\mathfrak{F}_{i}, \mathcal{H}_{i}\right) \bigwedge_{\mathcal{R}}(\mathfrak{L}, \mathcal{G})\right)=\left\{u_{1}, u_{4}, u_{5}, u_{6}, u_{7}\right\}$

therefore

$\kappa=\Gamma\left(\bigwedge_{i=1}^{2}\left(\mathfrak{F}_{i}, \mathcal{H}_{i}\right),(\mathfrak{L}, \mathcal{G})\right)=\frac{5}{8}=0.625$ which is same as of $\Gamma\left(\bigwedge_{i=1}^{3}\left(\mathfrak{F}_{i}, \mathcal{H}_{i}\right),(\mathfrak{L}, \mathcal{G})\right)$ calculated in Example 3.10. Hence $\left(\mathfrak{F}_{1}, \mathcal{H}_{1}\right) \cup\left(\mathfrak{F}_{2}, \mathcal{H}_{2}\right)$ is a reduct of $\mathbb{D}_{B H}$. 
Definition 19. Let $\mathbb{D}_{B H}=\left(\bigcup_{i=1}^{n}\left(\mathfrak{F}_{i}, \mathcal{H}_{i}\right),(\mathfrak{L}, \mathcal{G}), \mathfrak{U}\right)$. The significance of BHSS to decision hypersoft set, denoted $\Delta\left(\left(\mathfrak{F}_{j}, \mathcal{H}_{j}\right), \bigcup_{i=1}^{n}\left(\mathfrak{F}_{i}, \mathcal{H}_{i}\right),(\mathfrak{L}, \mathcal{G})\right)$, is defined as

$$
\Delta\left(\left(\mathfrak{F}_{j}, \mathcal{H}_{j}\right), \bigcup_{i=1}^{n}\left(\mathfrak{F}_{i}, \mathcal{H}_{i}\right),(\mathfrak{L}, \mathcal{G})\right)=\kappa-\Gamma((\mathfrak{P}, \mathcal{C}),(\mathfrak{L}, \mathcal{G}))
$$

where $(\mathfrak{P}, \mathcal{C})=\bigwedge_{i=1}^{n}\left(\mathfrak{F}_{i}, \mathcal{H}_{i}\right)(i \neq j)$.

Example 3.13. Since from Example 3.10, we have

$\kappa=\Gamma\left(\bigwedge_{i=1}^{3}\left(\mathfrak{F}_{i}, \mathcal{H}_{i}\right),(\mathfrak{L}, \mathcal{G})\right)=\frac{5}{8}=0.625$ and

$\left(\mathfrak{F}_{2}, \mathcal{H}_{2}\right) \bigwedge\left(\mathfrak{F}_{3}, \mathcal{H}_{3}\right)=\left\{\begin{array}{l}\left(\varepsilon_{1}=\left(\delta_{3}, \delta_{5}\right),\left\{u_{1}\right\}\right),\left(\varepsilon_{2}=\left(\delta_{3}, \delta_{6}\right),\left\{u_{5}, u_{6}\right\}\right),\left(\varepsilon_{3}=\left(\delta_{3}, \delta_{7}\right),\{\}\right) \\ \left.\left(\varepsilon_{4}=\left(\delta_{4}, \delta_{5}\right),\left\{u_{4}\right\}\right),\left(\varepsilon_{5}=\left(\delta_{4}, \delta_{6}\right),\{\}\right),\left(\varepsilon_{6}=\left(\delta_{4}, \delta_{7}\right),\left\{u_{7}, u_{8}\right\}\right)\right)\end{array}\right\}$.

$\left(\bigwedge_{i=2}^{3}\left(\mathfrak{F}_{i}, \mathcal{H}_{i}\right) \bigwedge_{\mathcal{R}}(\mathfrak{L}, \mathcal{G})\right)=\left\{u_{1}, u_{4}, u_{5}, u_{6}, u_{7}, u_{8}\right\}$

therefore

$\Gamma\left(\bigwedge_{i=2}^{3}\left(\mathfrak{F}_{i}, \mathcal{H}_{i}\right),(\mathfrak{L}, \mathcal{G})\right)=\frac{6}{8}=0.75$ hence

$\Delta\left(\left(\mathfrak{F}_{1}, \mathcal{H}_{1}\right), \underset{i=1}{\sim 3}\left(\mathfrak{F}_{i}, \mathcal{H}_{i}\right),(\mathfrak{L}, \mathcal{G})\right)=\kappa-\Gamma\left(\bigwedge_{i=2}^{3}\left(\mathfrak{F}_{i}, \mathcal{H}_{i}\right),(\mathfrak{L}, \mathcal{G})\right)=0.625-0.75=-0.125$

Definition 20. A BHSS $(\mathfrak{P}, \mathcal{C})$ is said to be a core BHSS of $\mathbb{D}_{B H}$ if it belongs to every reduct of $\mathbb{D}_{B H}$.

Definition 21. Let $\mathbb{D}_{B H}=((\mathfrak{F}, \mathcal{H}),(\mathfrak{L}, \mathcal{G}), \mathfrak{U})$, where $(\mathfrak{F}, \mathcal{H})=\bigcup_{i=1}^{n}\left(\mathfrak{F}_{i}, \mathcal{H}_{i}\right)$ and $\bigcup_{i=1}^{m}\left(\mathfrak{F}_{i}, \mathcal{H}_{i}\right) \subset$ $(\mathfrak{F}, \mathcal{H})$ is a reduct of $\mathbb{D}_{B H}$. Let $(\mathfrak{P}, \mathcal{C})=\bigwedge_{i=1}^{m}\left(\mathfrak{F}_{i}, \mathcal{H}_{i}\right)$. We say

$$
\text { if } e_{i} \text { then } e_{j}\left(\frac{\left|\mathfrak{P}\left(e_{i}\right)\right|}{\left|\mathfrak{L}\left(e_{j}\right)\right|}\right)
$$

a decision rule induced by $\bigcup_{i=1}^{m}\left(\mathfrak{F}_{i}, \mathcal{H}_{i}\right)$ where $e_{i} \in \mathcal{C}, \mathfrak{L}\left(e_{j}\right) \supseteq \mathfrak{P}\left(e_{i}\right), e_{j} \in \mathcal{G}$ and $\frac{\left|\mathfrak{P}\left(e_{i}\right)\right|}{\left|\mathfrak{L}\left(e_{j}\right)\right|}$ denotes the coverage proportion of rule.

Example 3.14. Since from Example 3.12, we have $\left(\mathfrak{F}_{1}, \mathcal{H}_{1}\right) \cup\left(\mathfrak{F}_{2}, \mathcal{H}_{2}\right)$ is a reduct of $\mathbb{D}_{B H}$ and

$$
\left(\mathfrak{F}_{1}, \mathcal{H}_{1}\right) \bigwedge\left(\mathfrak{F}_{2}, \mathcal{H}_{2}\right)=\left\{\begin{array}{l}
\left(\varepsilon_{1}=\left(\delta_{1}, \delta_{3}\right),\left\{u_{1}, u_{6}\right\}\right),\left(\varepsilon_{2}=\left(\delta_{1}, \delta_{4}\right),\left\{u_{4}\right\}\right), \\
\left.\left(\varepsilon_{3}=\left(\delta_{2}, \delta_{3}\right),\left\{u_{5}\right\}\right)\left(\varepsilon_{4}=\left(\delta_{2}, \delta_{4}\right),\left\{u_{7}\right\}\right)\right)
\end{array}\right\} .
$$

Now
(i) If $\varepsilon_{1}$ then $\delta_{8}(2 / 4)$
(ii) If $\varepsilon_{2}$ then $\delta_{8}(1 / 4)$
(iii) If $\varepsilon_{3}$ then $\delta_{8}(1 / 4)$
(iv) If $\varepsilon_{4}$ then $\delta_{9}(1 / 4)$ 


\section{An APPLICATION OF BIJECTIVE HYPERSOFT SET}

In this section, we present an application of bijective hypersoft set to establish decision rules which further help to have right decision regarding best selection of certain material/product. Suppose we have 16 kinds of laptops that form the universe of discourse $\mathfrak{U}=\left\{l_{1}, l_{2}, \ldots \ldots, l_{16}\right\}$. The most feasible laptop is expected to be selected by considering the attributes $a_{1}=$ Size, $a_{2}=$ Colour, $a_{3}=$ Processor, $a_{4}=$ RAM and $a_{5}=$ Price. The attribute-valued sets corresponding to these attributes are:

$\mathcal{A}=\left\{\right.$ small $=\alpha_{1}$, medium $=\alpha_{2}$, large $\left.=\alpha_{3}\right\}$

$\mathcal{B}=\left\{\right.$ Silver $=\beta_{1}$, Black $\left.=\beta_{2}\right\}$

$\mathcal{C}=\left\{2.60 \mathrm{GHz}=\gamma_{1}\right\}$

$\mathcal{D}=\left\{4.0 G B=\delta_{1}, 5.0 G B=\delta_{2}\right\}$

$\mathcal{E}=\left\{\right.$ normal $\left.=\varepsilon_{1}\right\}$.

Now we construct hypersoft set $(\Psi, \mathbb{J})$ where $\mathbb{J}=\mathcal{A} \times \mathcal{B} \times \mathcal{C} \times \mathcal{D} \times \mathcal{E}$

$$
\mathbb{J}=\left\{\begin{array}{l}
j_{1}=\left(\alpha_{1}, \beta_{1}, \gamma_{1}, \delta_{1}, \varepsilon_{1}\right), j_{2}=\left(\alpha_{1}, \beta_{1}, \gamma_{1}, \delta_{2}, \varepsilon_{1}\right), j_{3}=\left(\alpha_{1}, \beta_{2}, \gamma_{1}, \delta_{1}, \varepsilon_{1}\right), \\
j_{4}=\left(\alpha_{1}, \beta_{2}, \gamma_{1}, \delta_{2}, \varepsilon_{1}\right), j_{5}=\left(\alpha_{2}, \beta_{1}, \gamma_{1}, \delta_{1}, \varepsilon_{1}\right), j_{6}=\left(\alpha_{2}, \beta_{1}, \gamma_{1}, \delta_{2}, \varepsilon_{1}\right), \\
j_{7}=\left(\alpha_{2}, \beta_{2}, \gamma_{1}, \delta_{1}, \varepsilon_{1}\right), j_{8}=\left(\alpha_{2}, \beta_{2}, \gamma_{1}, \delta_{2}, \varepsilon_{1}\right), j_{9}=\left(\alpha_{3}, \beta_{1}, \gamma_{1}, \delta_{1}, \varepsilon_{1}\right), \\
j_{10}=\left(\alpha_{3}, \beta_{1}, \gamma_{1}, \delta_{2}, \varepsilon_{1}\right), j_{11}=\left(\alpha_{3}, \beta_{2}, \gamma_{1}, \delta_{1}, \varepsilon_{1}\right), j_{12}=\left(\alpha_{3}, \beta_{2}, \gamma_{1}, \delta_{2}, \varepsilon_{1}\right)
\end{array}\right\}
$$

and

$$
(\Psi, \mathbb{J})=\left\{\begin{array}{l}
\left(j_{1},\left\{l_{1}, l_{2}, l_{9}, l_{14}, l_{16}\right\}\right),\left(j_{2},\left\{l_{1}, l_{2}, l_{3}, l_{10}, l_{12}\right\}\right),\left(j_{3},\left\{l_{2}, l_{3}, l_{4}, l_{9}, l_{13}, l_{14}\right\}\right), \\
\left(j_{4},\left\{l_{4}, l_{5}, l_{6}, l_{10}, l_{15}, l_{16}\right\}\right),\left(j_{5},\left\{l_{6}, l_{7}, l_{8}, l_{10}, l_{12}\right\}\right),\left(j_{6},\left\{l_{2}, l_{3}, l_{4}, l_{7}, l_{9}\right\}\right), \\
\left(j_{7},\left\{l_{1}, l_{3}, l_{5}, l_{6}, l_{8}, l_{10}, l_{12}, l_{14}, l_{16}\right\}\right),\left(j_{8},\left\{l_{2}, l_{3}, l_{6}, l_{7}, l_{9}, l_{11}, l_{13}, l_{15}\right\}\right), \\
\left(j_{9},\left\{l_{5}, l_{7}, l_{8}, l_{9}, l_{11}, l_{13}, l_{16}\right\}\right),\left(j_{10},\left\{l_{2}, l_{4}, l_{6}, l_{8}, l_{10}, l_{12}, l_{14}\right\}\right), \\
\left(j_{11},\left\{l_{3}, l_{5}, l_{7}, l_{9}, l_{11}\right\}\right),\left(j_{12},\left\{l_{1}, l_{4}, l_{7}, l_{10}, l_{13}, l_{16}\right\}\right)
\end{array}\right\} .
$$

Now we propose an algorithm of bijective hypersoft sets to establish decision rules.

\section{ALGORITHM}

Step 1 Construct $\mathbb{D}_{B H}=\left(\bigcup_{i=1}^{n}\left(\mathcal{W}_{i}, \mathcal{V}_{i}\right),(\mathfrak{L}, \mathcal{J}), \mathfrak{U}\right)$

Step 2 Calculate $\kappa_{i}=\Gamma\left(\left(\mathcal{W}_{i}, \mathcal{V}_{i}\right),(\mathfrak{L}, \mathcal{J})\right)$ for $i=1,2, \ldots, n$

Step 3 Calculate $\kappa=\Gamma\left(\bigwedge_{j=1}^{n}\left(\mathcal{W}_{j}, \mathcal{V}_{j}\right),(\mathfrak{L}, \mathcal{J})\right)$

Step 4 Find reduct bijective hypersoft sets w.r.t. $\mathbb{D}_{B H}$

Step 5 Determine decision rules

[descriptive diagram] Step 1,Construct $\mathbb{D}_{B H}=\left(\cup_{i=1}^{n}\left(\mathcal{W}_{i}, \mathcal{V}_{i}\right),(\mathfrak{L}, \mathcal{J}), \mathfrak{U}\right)$, Step 2,

Calculate $\kappa_{i}=\Gamma\left(\left(\mathcal{W}_{i}, \mathcal{V}_{i}\right),(\mathfrak{L}, \mathcal{J})\right)$, Step 3, Calculate $\kappa=\Gamma\left(\bigwedge_{j=1}^{n}\left(\mathcal{W}_{j}, \mathcal{V}_{j}\right),(\mathfrak{L}, \mathcal{J})\right)$,

Step 4, Find reduct bijective hypersoft sets w.r.t. $\mathbb{D}_{B H}$, Step 5, Determine decision rules

\section{Step 1}

Let we have bijective hypersoft sets

$$
\left(\mathcal{W}_{1}, \mathcal{V}_{1}\right)=\left\{\left(j_{1},\left\{l_{1}, l_{4}, l_{6}\right\}\right),\left(j_{2},\left\{l_{2}, l_{5}, l_{7}\right\}\right)\right\}
$$




$$
\begin{gathered}
\left(\mathcal{W}_{2}, \mathcal{V}_{2}\right)=\left\{\left(j_{3},\left\{l_{1}, l_{5}, l_{6}\right\}\right),\left(j_{4},\left\{l_{4}, l_{7}, l_{8}\right\}\right)\right\} \\
\left(\mathcal{W}_{3}, \mathcal{V}_{3}\right)=\left\{\left(j_{5},\left\{l_{1}, l_{2}, l_{4}\right\}\right),\left(j_{6},\left\{l_{3}, l_{5}, l_{6}\right\}\right),\left(j_{7},\left\{l_{7}, l_{8}\right\}\right)\right\} \\
(\mathfrak{L}, \mathcal{J})=\left\{\left(j_{8},\left\{l_{1}, l_{4}, l_{5}, l_{6}\right\}\right),\left(j_{9},\left\{l_{2}, l_{3}, l_{7}, l_{8}\right\}\right)\right\}
\end{gathered}
$$

which form $\mathbb{D}_{B H}=\left(\cup_{i=1}^{3}\left(\mathcal{W}_{i}, \mathcal{V}_{i}\right),(\mathfrak{L}, \mathcal{J}), \mathfrak{U}\right)$ and $\mathcal{V}_{i}, \mathcal{J} \subseteq \mathbb{J}$.

\section{Step 2}

Since

$$
\left(\mathcal{W}_{1}, \mathcal{V}_{1}\right) \bigwedge\left(\mathcal{W}_{2}, \mathcal{V}_{2}\right)=\left\{\begin{array}{l}
\left(\theta_{1}=\left(j_{1}, j_{3}\right),\left\{l_{1}, l_{6}\right\}\right),\left(\theta_{2}=\left(j_{1}, j_{4}\right),\left\{l_{4}\right\}\right), \\
\left(\theta_{3}=\left(j_{2}, j_{3}\right),\left\{l_{5}\right\}\right)\left(\theta_{4}=\left(j_{2}, j_{4}\right),\left\{l_{7}\right\}\right)
\end{array}\right\}
$$

and

$\left(\mathcal{W}_{2}, \mathcal{V}_{2}\right) \bigwedge\left(\mathcal{W}_{3}, \mathcal{V}_{3}\right)=\left\{\begin{array}{l}\left(\theta_{5}=\left(j_{3}, j_{5}\right),\left\{l_{1}\right\}\right),\left(\theta_{6}=\left(j_{3}, j_{6}\right),\left\{l_{5}, l_{6}\right\}\right),\left(\theta_{7}=\left(j_{3}, j_{7}\right), \emptyset\right) \\ \left(\theta_{8}=\left(j_{4}, j_{5}\right),\left\{l_{4}\right\}\right),\left(\theta_{9}=\left(j_{4}, j_{6}\right), \emptyset\right),\left(\theta_{10}=\left(j_{4}, j_{7}\right),\left\{l_{7}, l_{8}\right\}\right)\end{array}\right\}$

and

$\left(\mathcal{W}_{1}, \mathcal{V}_{1}\right) \bigwedge\left(\mathcal{W}_{3}, \mathcal{V}_{3}\right)=\left\{\begin{array}{l}\left(\theta_{11}=\left(j_{1}, j_{5}\right),\left\{l_{1}, l_{4}\right\}\right),\left(\theta_{12}=\left(j_{1}, j_{6}\right),\left\{l_{6}\right\}\right),\left(\theta_{13}=\left(j_{1}, j_{7}\right), \emptyset\right) \\ \left(\theta_{14}=\left(j_{2}, j_{5}\right),\left\{l_{2}\right\}\right),\left(\theta_{15}=\left(j_{2}, j_{6}\right),\left\{l_{5}\right\}\right),\left(\theta_{16}=\left(j_{2}, j_{7}\right),\left\{l_{7}\right\}\right)\end{array}\right\}$

Now

$$
\begin{aligned}
& \kappa_{1}=\Gamma\left(\left(\mathcal{W}_{1}, \mathcal{V}_{1}\right),(\mathfrak{L}, \mathcal{J})\right)=\frac{3}{16}=0.1875 \\
& \kappa_{2}=\Gamma\left(\left(\mathcal{W}_{2}, \mathcal{V}_{2}\right),(\mathfrak{L}, \mathcal{J})\right)=\frac{3}{16}=0.1875 \\
& \kappa_{3}=\Gamma\left(\left(\mathcal{W}_{3}, \mathcal{V}_{3}\right),(\mathfrak{L}, \mathcal{J})\right)=\frac{2}{16}=0.125 \\
& \kappa_{4}=\Gamma\left(\left(\mathcal{W}_{1}, \mathcal{V}_{1}\right) \bigwedge\left(\mathcal{W}_{2}, \mathcal{V}_{2}\right),(\mathfrak{L}, \mathcal{J})\right)=\frac{5}{16}=0.3125 \\
& \kappa_{5}=\Gamma\left(\left(\mathcal{W}_{2}, \mathcal{V}_{2}\right) \bigwedge\left(\mathcal{W}_{3}, \mathcal{V}_{3}\right),(\mathfrak{L}, \mathcal{J})\right)=\frac{6}{16}=0.375 \\
& \kappa_{6}=\Gamma\left(\left(\mathcal{W}_{1}, \mathcal{V}_{1}\right) \bigwedge\left(\mathcal{W}_{3}, \mathcal{V}_{3}\right),(\mathfrak{L}, \mathcal{J})\right)=\frac{6}{16}=0.375
\end{aligned}
$$

\section{Step 3}

$\left(\mathcal{W}_{1}, \mathcal{V}_{1}\right) \wedge\left(\mathcal{W}_{2}, \mathcal{V}_{2}\right) \wedge\left(\mathcal{W}_{3}, \mathcal{V}_{3}\right)=$

$$
\left\{\begin{array}{c}
\left(\eta_{1}=\left(j_{1}, j_{3}, j_{5}\right),\left\{l_{1}\right\}\right),\left(\eta_{2}=\left(j_{1}, j_{3}, j_{6}\right),\left\{l_{6}\right\}\right),\left(\eta_{3}=\left(j_{1}, j_{3}, j_{7}\right), \emptyset\right) \\
\left(\eta_{4}=\left(j_{1}, j_{4}, j_{5}\right),\left\{l_{4}\right\}\right),\left(\eta_{5}=\left(j_{1}, j_{4}, j_{6}\right), \emptyset\right),\left(\eta_{6}=\left(j_{1}, j_{4}, j_{7}\right), \emptyset\right) \\
\left(\eta_{7}=\left(j_{2}, j_{3}, j_{5}\right), \emptyset\right),\left(\eta_{8}=\left(j_{2}, j_{3}, j_{6}\right),\left\{l_{5}\right\}\right),\left(\eta_{9}=\left(j_{2}, j_{3}, j_{7}\right), \emptyset\right) \\
\left(\eta_{10}=\left(j_{2}, j_{4}, j_{5}\right), \emptyset\right),\left(\eta_{11}=\left(j_{2}, j_{4}, j_{6}\right), \emptyset\right),\left(\eta_{12}=\left(j_{2}, j_{4}, j_{7}\right),\left\{l_{7}\right\}\right)
\end{array}\right\} .
$$

therefore

$$
\kappa=\Gamma\left(\bigwedge_{i=1}^{3}\left(\mathcal{W}_{i}, \mathcal{V}_{i}\right),(\mathfrak{L}, \mathcal{J})\right)=\frac{5}{16}=0.3125
$$

\section{Step 4}

As

$$
\Gamma\left(\left(\mathcal{W}_{1}, \mathcal{V}_{1}\right) \bigwedge\left(\mathcal{W}_{2}, \mathcal{V}_{2}\right),(\mathfrak{L}, \mathcal{J})\right)=0.3125=\Gamma\left(\bigwedge_{i=1}^{3}\left(\mathcal{W}_{i}, \mathcal{V}_{i}\right),(\mathfrak{L}, \mathcal{J})\right)
$$

therefore $\left(\mathcal{W}_{1}, \mathcal{V}_{1}\right) \cup\left(\mathcal{W}_{2}, \mathcal{V}_{2}\right)$ is a reduct of $\mathbb{D}_{B H}$.

\section{Step 5}

Since $\left(\mathcal{W}_{1}, \mathcal{V}_{1}\right) \cup\left(\mathcal{W}_{2}, \mathcal{V}_{2}\right)$ is a reduct of $\mathbb{D}_{B H}$ therefore we have the following decision rules w.r.t. $\mathbb{D}_{B H}$

(i) If $\theta_{1}$ then $j_{8}(2 / 4)$

(ii) If $\theta_{2}$ then $j_{8}(1 / 4)$ 
(iii) If $\theta_{3}$ then $j_{8}(1 / 4)$

(iv) If $\theta_{4}$ then $j_{9}(1 / 4)$

Hence we have two elements $j_{8}$ and $j_{9}$ in our decision set and $j_{8}$ is more preferable than $j_{9}$ in this set for further evaluation.

4.1. Comparative study. In this subsection, we compare our propose structure with the existing studies.

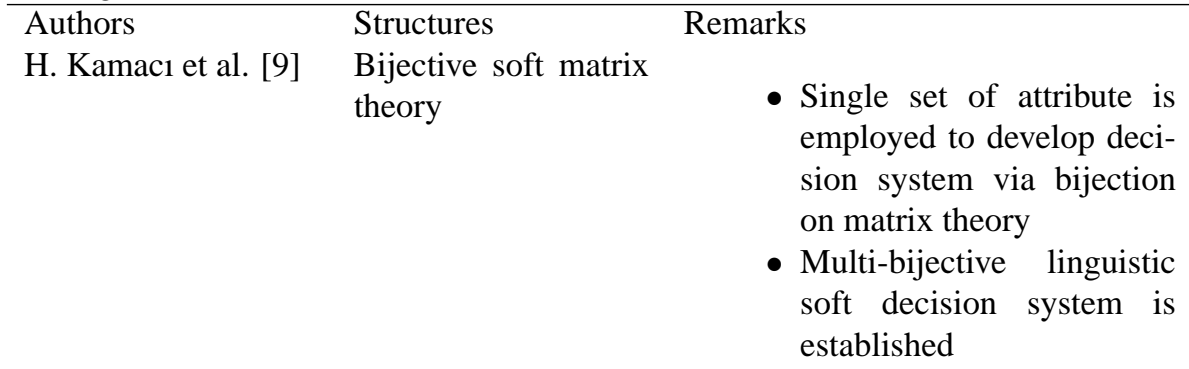

Gong et al. [7] The bijective soft set

- Single set of attribute is employed to develop decision system

$\begin{array}{lll}\text { Proposed structure } & \text { Bijective hypersoft } & \text { - Attributes are further } \\ \text { set } & \text { classified into disjoint } \\ & \text { attribute-valued sets } \\ & \text { - Decision system is de- } \\ & \text { veloped via employment } \\ \text { of multi-argument approx- } & \text { imate functions. }\end{array}$

\section{CONCLUSION}

In this study, the concept of bijective hypersoft set is developed and its some set aggregation operations such as restricted-AND and relaxed-AND, are discussed. Decision making algorithm and its application are discussed with the support of new proposed operations of bijective hypersoft set like dependency, decision system, significance of decision system, reduced decision system and decision rules in decision system. In order to adequate the existing soft-like structures for multi-argument functions, future work may include the hybridized study of proposed work with the following structures:

- Fuzzy Set

- Intuitionistic Fuzzy Set

- Interval-Valued Fuzzy Set

- Pythagorean Fuzzy Set

- q-rung Orthopair Fuzzy Set

- Rough Set 
- Complex Set

- Neutrosophic Set etc.

and their applications in decision making.

\section{REFERENCES}

[1] M. Akram, G. Ali, J.C.R. Alcantud, New decision-making hybrid model : intuitionistic fuzzy N-soft rough sets, Soft Computing, 23, No.20 (2019) 9853-9868.

[2] M.I. Ali, F. Feng, X. Liu, W.K. Min, M. Sabir, On some new operations in soft set theory, Computers and Mathematics with Applications 57 (2009) 1547-1553.

[3] K.V. Babitha, J.J. Sunil, Soft set relations and functions, Computers and Mathematics with Applications 60 (2010) 1840-1849.

[4] K.V. Babitha, J.J. Sunil, Transitive closure and ordering in soft set, Computers and Mathematics with Applications 61 (2011) 2235-2239.

[5] F. Feng, C. Li, B. Davvaz, M.I. Ali, Soft sets combined with fuzzy sets and rough sets : a tentative approach, Soft Computing 14, No.9 (2010) 899-911.

[6] X. Ge, S. Yang, Investigations on some operations of soft sets, World Academy of Science Engineering and Technology 75 (2011) 1113-1116.

[7] K. Gong, X. Zhi, Z. Xia, The bijective soft set with its operations, Computers Mathematics with Applications 60, No. 8 (2010) 2270-2278.

[8] M. Ihsan, A.U. Rahman, M. Saeed, Hypersoft Expert Set With Application in Decision Making for Recruitment Process, Neutrosophic Sets and Systems 42 (2021) 191-207.

[9] H. Kamac1, A.O. Atagün, E. Toktaş, Bijective soft matrix theory and multi-bijective linguistic soft decision system, Filomat, 32, No.11 (2018) 3799-3814

[10] H. Kamac1, A.O. Atagün, E. Aygün, Difference operations of soft matrices with applications in decision making, Punjab Uni. j. math. 51, No.3 (2019) 1-21.

[11] H. Kamac1, Introduction to $N$-soft algebraic structures, Turkish Journal of Mathematics, 44, No.6 (2020) 2356-2379.

[12] H. Kamac1, S. Petchimuthu, Bipolar N-soft set theory with applications, Soft Computing, 24, No.22 (2020) 16727-16743.

[13] H. Kamac1, On hybrid structures of hypersoft sets and rough sets, International Journal of Modern Science and Technology, 6, No.4 (2021) 69-82.

[14] A. Khalid, M. Abbas, Distance measures and operations in intuitionistic and interval-valued intuitionistic fuzzy soft set theory, International Journal of Fuzzy Systems, 17, No.3 (2005) 490-497.

[15] A.Z. Khameneh, A. Kilicman, Parameter reduction of fuzzy soft sets : An adjustable approach based on the three-way decision, International Journal of Fuzzy Systems, 20, No.3 (2018) 928-942.

[16] F. Li, Notes on soft set operations, ARPN Journal of systems and softwares 1, No.6 (2011) 205-208.

[17] P.K. Maji, R. Biswas, A.R. Roy, Soft Set Theory, Computers and Mathematics with Applications, 45, (2003) 555-562.

[18] N. Martin, F. Smarandache, Concentric Plithogenic Hypergraph based on Plithogenic Hypersoft Sets- A Novel Outlook, Neutrosophic Sets and Systems, 33, (2020) 78-91.

[19] D. Molodtsov, Soft Set Theory - First Results, Computers and Mathematics with Applications, 37, (1999) 19-31.

[20] T.Y. Ozturk, A. Yolcu, On Neutrosophic Hypersoft Topological spaces, Theory and Application of Hypersoft Set, Pons Publishing House, Brussel, (2021) 215-234.

[21] B. Paik, S.K. Mondal, A distance-similarity method to solve fuzzy sets and fuzzy soft sets based decisionmaking problems, Soft Computing, 24, No.7 (2020) 5217-5229.

[22] D. Pei, D. Miao, From soft set to information system, In international conference of granular computing, IEEE, 2, (2005) 617-621.

[23] S. Petchimuthu, H. Kamac1, The row-products of inverse soft matrices in multicriteria decision making, Journal of Intelligent and Fuzzy Systems, 36, No. 6 (2019) 6425-6441. 
[24] A.U. Rahman, M. Saeed, F. Smarandache, M.R. Ahmad, Development of Hybrids of Hypersoft Set with Complex Fuzzy Set, Complex Intuitionistic Fuzzy set and Complex Neutrosophic Set, Neutrosophic Sets and Systems, 38, (2020) 335-354.

[25] A.U. Rahman, M. Saeed, F. Smarandache, Convex and Concave Hypersoft Sets with Some Properties, Neutrosophic Sets and Systems, 38, (2020) 497-508.

[26] A.U. Rahman, M. Saeed, A. Dhital, Decision Making Application Based on Neutrosophic Parameterized Hypersoft Set Theory, Neutrosophic Sets and Systems, 41, (2021) 1-14.

[27] A.U. Rahman, M. Saeed, S. Zahid, Application in Decision Making Based on Fuzzy Parameterized Hypersoft Set Theory, Asia Mathematika, 5, No.1 (2021) 19-27.

[28] A.U. Rahman, A. Hafeez, M. Saeed, M.R. Ahmad, U. Farwa, Development of Rough Hypersoft Set with Application in Decision Making for the Best Choice of Chemical Material, In Theory and Application of Hypersoft Set, Pons Publication House, Brussel, (2021) 192-202.

[29] M. Saeed, M. Hussain, A.A. Mughal, A Study of Soft Sets with Soft Members and Soft Elements: A New Approach, Punjab Univ. j.math.52, No.8 (2020) 1-15.

[30] M. Saeed, M. Ahsan, M.K. Siddique, A.R. Ahmad, A Study of the Fundamentals of Hypersoft Set Theory, International Journal of Scientific and Engineering Research, 11, No.1 (2020) 320-329.

[31] M. Saeed, A.U. Rahman, M. Ahsan, F. Smarandache, An Inclusive Study on Fundamentals of Hypersoft Set, In Theory and Application of Hypersoft Set, Pons Publishing House, Brussel, (2021) 1-23.

[32] M. Saeed, M. Ahsan, A.U. Rahman, A novel approach to mappings on hypersoft classes with application, In Theory and Application of Hypersoft Set, Pons Publication House, Brussel, (2021) 175-191.

[33] M. Saeed, M. Ahsan, M. H. Saeed, A. Mehmood, T. Abdeljawad, An Application of Neutrosophic Hypersoft Mapping to Diagnose Hepatitis and Propose Appropriate Treatment, IEEE Access, 9, (2021) 70455-70471.

[34] M. Saeed, M. Ahsan, T. Abdeljawad, A Development of Complex Multi-Fuzzy Hypersoft Set With Application in MCDM Based on Entropy and Similarity Measure, IEEE Access, 9, (2021) 60026-60042.

[35] M. Saeed, M.K. Siddique, M. Ahsan, M.R. Ahmad, A.U. Rahman, A Novel Approach to the Rudiments of Hypersoft Graphs, In Theory and Application of Hypersoft Set, Pons Publication House, Brussel, (2021) 203-214.

[36] M. Saqlain, N. Jafar, S. Moin, M. Saeed, S. Broumi, Single and Multi-valued Neutrosophic Hypersoft Set and Tangent Similarity Measure of Single valued Neutrosophic Hypersoft Sets, Neutrosophic Sets and Systems, 32, (2020) 317-329.

[37] M. Saqlain, S. Moin, N. Jafar, M. Saeed, F. Smarandache, Aggregate Operators of Neutrosophic Hypersoft Sets, Neutrosophic Sets and Systems, 32, (2020) 294-306.

[38] M. Saqlain, M. Saeed, M.R. Ahmad, F. Smarandache, Generalization of TOPSIS for Neutrosophic Hypersoft Sets using Accuracy Function and its Application, Neutrosophic Sets and Systems, 27, (2019) 131-137.

[39] A. Sezgin, A.O. Atagün, On operations of soft sets, Computers and Mathematics with Applications, 61, No.5 (2011) 1457-1467.

[40] F. Smarandache, Extension of Soft Set of Hypersoft Set, and then to Plithogenic Hypersoft Set, Neutrosophic Sets and Systems 22 (2018) 168-170.

[41] A. Yolcu, T.Y. Ozturk, Fuzzy Hypersoft Sets and Its Application to Decision-Making, In Theory and Application of Hypersoft Set, Pons Publishing House, Brussel, (2021) 50-64.

[42] A. Yolcu, F. Smarandache, T.Y. Ozturk, Intuitionistic fuzzy hypersoft sets, Communications Faculty of Sciences University of Ankara Series A1 Mathematics and Statistics, 70, No.1 (2021) 443-455.

[43] J. Zhang, X. Wu, R. Lu, Decision Analysis Methods Combining Quantitative Logic and Fuzzy Soft Sets, International Journal of Fuzzy Systems, 22, No.6 (2020) 1801-1814. 